\title{
THE CRITICAL ROTATION OF STRANGE STARS AND RAPIDLY ROTATING PULSARS
}

\author{
Zheng Xiaoping and Yang Shuhua \\ Department of Physics, Huazhong Normal University, Wuhan 430079 \\ zhxp@phy.ccnu.edu.cn \\ Li Jiarong \\ The Institute of Particle Physics, Huazhong Normal University, Wuhan 430079
}

\begin{abstract}
We utilize the bulk viscosity of interacting strange quark matter to reevaluate the damping time scale. The presence of medium effect of bulk viscosity leads to a stronger damping of rmodes, which can be over an order of magnitude for realistic parameters. We find that the r-mode instability window is narrowed due to the medium effect, and hence when a pulsar reaches the instability window it will only slow down by gravitational wave emission to a period of $1.78 \mathrm{msec}$ instead of $2.5 \mathrm{msec}$ given by early estimate. As a theoretical upper rotation limit of pulsars, the period of $1.78 \mathrm{msec}$ is very close to the two most rapidly spinning pulsars known, with periods of about $1.6 \mathrm{msec}$.

PACS numbers: 97.60.Jd,12.38.Mh, 97.60.Gb
\end{abstract}

Subject headings: dense matter-gravitation-stars:strange-stars:rotation

\section{INTRODUCTION}

At high density, normal hadron matter has been predicted a deconfined transition, where quarkgluon plasma is formed. This could have important consequences for compact stars, with central densities several times the nuclear saturation density. Ever since strange quark matter(SQM), a conglomerate of up, down and strange quarks, has been suggested as a possible absolutely stable or metastable phase of nuclear matter(Witten 1984), it has been speculated that strange stars might exist in the universe(Alcock et al. 1986; Haensel et al. 1986; Colpi and Miller 1992).

The studies that quark interactions within lowest order perturbative QCD were considered in the MIT bag model also predicted that sufficiently heavy strangelets might be absolutely stable(Fahari and Jaffe 1984). If the SQM hypothesis is correct then some (perhaps all) pulsars may be strange stars(Alcock et al. 1986; Haensel et al.
1986; Colpi and Miller 1992). Thus it would have obvious implications for our understanding of pulsars if strange stars could exist. It is an important subject that probing possible observational evidences of the existence of strange stars by astrophysical investigations.

Since self-bound of SQM due to strong interaction is very important, the mass-radius relation of the assumed strange stars has been uncovered to be different from that of neutron stars. However, for the canonical mass of $1.4 M_{\odot}$, gravity dominates the strong interaction which leads to strange stars and neutrons being similar in size(Alcock et al. 1986). Therefore, the attempt to distinguish strange stars from neutron stars for given observed masses and radii of pulsars seems to have encountered with great difficulties. Strange stars could indicate the distinguishable signal from neutron stars by their cooling properties, but the difference in the cooling behavior of strange stars and neutron stars will disappears if the direct Urca process 
is operating in the core of an neutron star, which is done either by the proton fraction rising above $10 \%$ (Lattimer et al. 1994) or by hyperons being present in the core of a neutron star(Page et al. 2000). This would make it difficult to identify a strange star from cooling data.

Ever since Andersson realized that the r-modes are unstable at all rates of rotation in perfect fluid stars(Andersson 1998), a series of papers have investigated the many implications for gravitational radiation detection and the evolution of pulsar(Friedman and Morsink 1998; Lindblom et al. 1998; Owen et al. 1998; Kojima 1998; Madsen 1998; Andersson et al. 1999a,b; Ho 2000; Rezania and Maartens 2000; Madsen 2000; Andersson et al. 2001). In recent years, several crucial issues regarding the astrophysical relevance of the r-mode instability have been investigated. Key results concern the interaction between oscillations in core fluid and the crust(Bildsten \& Ushomirsky 2000; Andersson et al. 2000; Lindblom et al. 2000), the role of the magnetic field(Spruit 1999; Rezzolla et al. 2000; Mendell 2001) superfluidity(Lindblom \& Mendell 2000; Andersson and Comer 2001) and the effect of exotic particles that are thought to exist in the deep neutron star core(Jones 2001; Lindblom et al. 2001).

Meanwhile, Madsen (1998) has pointed out that the r-mode instability may provide the means to distinguish strange stars from neutron stars. The main reason for this is that the viscosity coefficients are rather different in these two cases. While the shear viscosity of a strange star is comparable to that of a neutron star, the bulk viscosity would be many orders of magnitude stronger than its neutron star counterpart. This has interesting effects on the r-mode instability. Based on the characteristic r-mode instability window of strange stars, which is related to the gravitational wave emission and the viscosity, a few mechanisms have been discussed to explain the clustering of spin-frequencies of LMXBs(Madsen 2000; Andersson et al. 2001).

However, the bulk viscosity coefficient in Madsen's studies takes the one for the case of noninteracting quark gas in MIT bag(Madsen 1992). In fact, if the interaction among quarks is considered, the bulk viscosity increases, which is calculated by Zheng et al. (2002). As we will show in the following, the r-mode instability window for strange stars will be significantly modified, when the improved bulk viscosity is adopted.

As demonstrated blow, we find the r-mode instability window is evidently narrowed due to the inclusion of medium effect(interactions among constituent particles). The medium effect makes the lowest critical spin frequency rise to $558 \mathrm{~Hz}$ from about $300-400 \mathrm{~Hz}$ (for the case of free quark gas). The limiting period of $1.78 \mathrm{msec}$ corresponding $558 \mathrm{~Hz}$ is closer to the two most rapidly spinning pulsars known (with the periods of $1.56 \mathrm{msec}$ and $1.61 \mathrm{msec}$ ) than the periods of $2.5 \mathrm{msec}$ and 3 msec given by Madsen.

In addition, if it could be established that rapidly spinning pulsar are strange stars, the pulsar data would put certain constraints on the model parameter, namely the current mass of strange quark mass $m_{s}$. As we can see in Madsen's article(Madsen 2000), in the normal and especially in the 2-flavor color superconducting phase (2SC) case, the theoretical results is not consistent with the pulsar data when $m_{s}=100 \mathrm{MeV}$. However, when we taking the medium effects into account, the constraints to $m_{s}$ from pulsar data appears to be deeply relaxed, and we will show that $m_{s}$ can be taken as $100 \mathrm{MeV}$ in this article.

This paper is organized as the following. In section 2, we recall the bulk viscosity of interacting quark matter and compute the damping time scale on r-modes. In section 3, we give the improved critical curve(spin frequency) in spin frequency $(\nu)$-temperature $(T)$ plane. In section 4 , we give a summary.

\section{BULK VISCOSITY AND DISSIPA- TION ON r-MODES}

Ever since Wang \& Lu (1984) pointed out that strange quark matter is characterized by a huge bulk viscosity relative to nuclear matter, some investigations have tried to calculate the relevant viscosity coefficient of SQM(Sawyer 1989; Madsen 1992; Goyal et al. 1994). In a MIT bag model, it is thought that the exact solution had been obtained by Madsen(Madsen 1992). However, he ignored the coupling among quarks in the bag, which had been considered in the study of the equation of state of SQM(Schertler et al. 1997). Soon before, we found the coupling's effect on the bulk viscosity leads to an increase of the viscosity over an 
order of magnitude although the medium modifications of the equation of state of strange quark matter was proven to be small(Zheng et al. 2002). To very good approximation, the relevant bulk viscosity coefficient still takes the form formulated by Madsen (1992):

$$
\zeta=\frac{\alpha T^{2}}{\omega^{2}+\beta T^{4}}
$$

but here $\alpha$ and $\beta$ were given by (Zheng et al. 2002), which are extremely differing from Madsen's and strongly depending on the coupling constant of strong interactions among quarks. We expressed them as

$$
\begin{gathered}
\alpha=9.39 \times 10^{22} \mu_{d}^{5}\left(\frac{k_{\mathrm{F} d}^{2}}{C_{d}}-\frac{k_{\mathrm{F} s}^{2}}{C_{s}}\right)^{2}\left(\mathrm{gcm}^{-1} \mathrm{~s}^{-1}\right), \\
\beta=7.11 \times 14^{-4}\left[\frac{\mu_{d}^{5}}{2}\left(\frac{1}{k_{\mathrm{F} d} C_{d}}-\frac{1}{k_{\mathrm{F} s} C_{s}}\right)\right]^{2}\left(\mathrm{~s}^{-2}\right),
\end{gathered}
$$

where $k_{F i}=\left(\mu_{i}^{2}-m_{i}^{* 2}\right)^{1 / 2}, C_{i}=\mu_{i}-m_{i}^{*} \frac{\partial m_{i}^{*}}{\partial \mu_{i}}$ and $m_{i}^{*}$ was given in the references(Klimov 1982; Weldon 1993; Pisarski 1989; Blaizot and Ollitrault 1993; Vija and Thoma 1995).

The bulk viscosity is expected to be the dominated internal fluid mechanism in hot compact stars. The timescales for the bulk viscosity damping of r-modes need to be estimated. Since the coupling of the r-modes to bulk viscosity vanishes in the lowest-order expression, the derived timescales must be based on the fully self-consistent second-order calculation of this coupling. The earlier estimates can't gain this end(Lindblom et al. 1998; Andersson et al. 1999a; Kokkotas and Stergioulas 1999). But it has been completed by Lindblom et al. (1999). We substitute our viscosity for the given Low-T $\operatorname{limit}\left(T<10^{9} \mathrm{~K}\right)$ into the formula $(6.2)$ given by Lindblom et al. (1999), and find the only difference comparing to the early calculation is the change of the viscosity coefficient. Thus, we can immediately obtain the timescale by a simple comparison instead of the repeat of the complicated calculations

$$
\tau_{B}=\bar{\tau}_{B} \mathrm{~S}\left(\pi G \bar{\rho} / \Omega^{2}\right) T_{9}^{-2}
$$

with

$$
\bar{\tau}_{B}=2.83 \times 10^{3} \alpha^{-1} \bar{\rho} m_{s 100}^{4} .
$$

where $T_{9}$ and $m_{100}$ denote temperature in units of $10^{9} \mathrm{~K}$ and the current mass of strange quark in units of $100 \mathrm{MeV}$, and $\bar{\rho}$ is the mean density of the star.

Evidently, $\bar{\tau}_{B}$ is determined with the chemical potential $\mu_{d}$, which can be obtained by solving the equations related to chemical equilibrium, electric charge neutrality and conservation of baryon number for given $\bar{\rho}$, namely

$$
\begin{gathered}
\mu_{s}=\mu_{d}, \mu_{u}=\mu-\mu_{e} \\
\frac{2}{3} n_{u}-\frac{1}{3}\left(n_{d}+n_{s}\right)-n_{e}=0 \\
n=\frac{1}{3}\left(n_{u}+n_{d}+n_{s}\right) \\
\bar{\rho}=\left(\frac{E}{A}\right) n
\end{gathered}
$$

Here, $n$ denotes the baryon number density, and $n_{i}=\frac{1}{6 \pi^{2}} k_{F i}^{2}$ is the particle number density. $\frac{E}{A}$ is the energy per baryon and we approximately take it as the mass of a neutron in our calculation.

The coupling $g$ will extremely influences the solutions of the above equations. Following Schertler et al. (1997), $g$ is taken as a free parameter ranging from 0 to 5 and the equations $(6)$ (7) (8) are solved numerically. Figure 1 shows $\bar{\tau}_{B}$ as a function of $\bar{\rho}$ for different $g$ and $m_{s}=200 \mathrm{MeV}$. The time scales depend on $g$ remarkably while they are nearly independent of $\bar{\rho}$. The time scale for $g=5$ arrives at tens of times shorter than the case of $g=0$. For a strange star with mass $M=1.4 M_{\odot}$ and radius $R=10 \mathrm{~km}$, we find $\bar{\tau}_{B}$ ranges from $4.24 \times 10^{-2} \mathrm{~s}(g=0)$ to $1.53 \times 10^{-3} \mathrm{~s}(g=5)$. This is of great interest because this will significantly increases the critical rotation angular velocity for the onset of r-mode instability.

\section{CRITICAL SPIN FREQUENCY FOR THE ONSET OF THE r-MODE IN- STABILITY}

The r-mode unstable (or stable) regime of the relativistic stars, neutron stars as well as strange stars, depends on the competition between the gravitational radiation and various dissipation mechanisms. To plot the instability window of r-mode or gain the critical rotation frequency for a given stellar model as a function of temperature, we need to acquire the characteristic timescales, 
damping and growing timescales of r-mode instability. Due to the emission of gravitational waves, the r-mode grows on a timescale

$$
\tau_{\mathrm{G}}=\bar{\tau}_{\mathrm{G}}\left(\pi G \bar{\rho} / \Omega^{2}\right)^{3}
$$

where $\bar{\tau}_{\mathrm{G}}$ is $-3.26(1.57) \mathrm{s}$ for $n=1(0)$ polytropic EOS, which was respectively studied by Lindblom et al. (1999) and Kokkotas and Stergioulas (1999). For the viscous damping timescales, we shall also consider the shear viscosity besides bulk viscosity discussed in the last section. In strange stars, the time scale for the shear viscous damping is given

$$
\tau_{S}=\bar{\tau}_{S}\left(\alpha_{S} / 0.1\right)^{5 / 3} T_{9}^{5 / 3}
$$

Here, $\bar{\tau}_{S}$ is $5.37(2.40) \times 10^{8} \mathrm{~s}$ corresponding to $n=$ $1(0), \alpha_{S}$ is the strong coupling and we take $\alpha_{S}=$ 0.1 in following calculation(Madsen 2000).

We can now evaluate the critical spin frequency as a function of temperature from the equation

$$
\frac{1}{\tau_{G}}+\frac{1}{\tau_{S}}+\frac{1}{\tau_{B}}=0
$$

Figure 2 shows the regions of r-mode (in)stability in spin frequency-temperature $(\nu-T)$ plane for a strange star with mass $M=1.4 M_{\odot}$ and radius $R=10 \mathrm{~km}$. The shading between the two curves displays the effect of the medium modification of quark masses on critical rotation frequencies. The medium effect narrows the r-mode instability window. The dotted curve corresponds to $g=0$, reduced to the Madsen's result(Madsen 2000), and $g=5$ is assumed in the upper contour(the solid curve). The upper contour has a lowest limiting frequency denoted by $C$ as $558 \mathrm{~Hz}$ (the corresponding period is $1.78 \mathrm{msec}$ ), which is more close to the two most rapidly spinning pulsars known, with frequencies of $642 \mathrm{~Hz}$ and $622 \mathrm{~Hz}$ ( the periods are 1.56 and $1.61 \mathrm{msec}$ ), than the period of $2.5 \mathrm{msec}$. This implies a strange star would slow down by gravitational wave emission when it reaches the instability window and spin around in $1.78 \mathrm{msec}$ instead of the $2.5 \sim 3 \mathrm{msec}$ expected by Madsen. Figure 3 shows the results for 2SC stars. Similar to figure 1 , the medium effect also increases the critical rotation frequency of 2SC stars, but it should be stressed that the medium effect leaves the most rapidly stars away from the instability window.
Figure 4 depicts the instability windows where the current mass of strange quark takes $100 \mathrm{MeV}$. If the rapidly rotation pulsars could be regarded as strange stars, the medium effect would relax the stringent constraint on the choice of QCD parameters in contrast to the non-interacting medium case, for example, the 2SC stars for smaller current mass $m_{s}$ can safely exist due to the medium effect(see the upper dashed curve in figure 4).

\section{CONCLUSIONS AND DISCUSSIONS}

We apply the bulk viscous coefficient including medium effect to reevaluate and discuss the viscous damping time. The time greatly depends on the strong coupling $g$. We find the time scale $\bar{\tau}_{B}$, rather than non-interacting perfect fluid where $\bar{\tau}_{B}$ is constant(The fact is that $\bar{\tau}_{B}$ versus $\bar{\rho}$ slowly increases for non-vanishing $m_{s}$ as shown in figure 1 ), weakly decreases with increasing mass density of stars for the given larger $g$. Therefore, the medium effect due to strong interactions among quarks adds the viscous dissipation of instability modes. For a star with $M=1.4 M_{\odot}$ and radius $R=10 \mathrm{~km}$, we numerically calculate the critical spin frequency as a function of temperature. The medium effect lead to a significant lift of the critical frequencies. We find that the largest critical period is reduced to $1.78 \mathrm{msec}$ from $2.5 \mathrm{msec}$, appearing to be more close to the two most rapidly spinning pulsars known.

We also study the 2SC stars and give our improved scenario. The current mass of strange quark in the model have a broader range taking the medium effects into account.

Finally, it should be mentioned that we here only consider weak coupling of strong interactions among quarks in MIT bag. The long range unperturbation interactions are contained in the usual bag constant. Although the MIT bag constant is able to contribute to EOS of SQM, the long range effects on dynamical quantities, such as viscous coefficient etc., seem to be ignored due to the hiding of the microscopic processes into the bag constant. If the processes can be taken into account, we may conjecture that the limiting spin of strange stars would shift further upward. This is our future work. 


\section{Acknowledgments}

We would like to thank the partial support by National Natural Science Foundation of China under Grant No. 10175026 and Natural Science Foundation of CCNU.

\section{REFERENCES}

Alcock C., Farli E. and Olinto R., 1986, ApJ, 310, 261.

Andersson N., 1998, ApJ, 502, 708

Andersson N., Kokkotas K.D. and Schutz B.F., 1999, ApJ, 510, 846

Andersson N., Kokkotas K.D. and Stergioulas N., 1999, ApJ, 516, 307.

Andersson N., Jones D.I., Kokkotas K.D. and Stergioulas N., 2000, ApJ, 534, L75.

Andersson N. and Comer G.L., 2001, MNRAS, $328,1129$.

Andersson N., Jones D.I. and Kokkotas K.D. [ astro-ph/0111582].

Bildsten L. and Ushomirsky G., 2000, ApJ, 529, 33

Blaizot J.-P. and Ollitrault J.-Y., 1993, Phys. Rev. D, 48, 1390.

Colpi M. and Miller J.C., 1992, ApJ, 388, 513.

Fahri E. and Jaffe R.L., 1984, Phys. Rev. D, 30, 2379 .

Friedman J.L. and Morsink S.M., 1998, ApJ, 502, 714.

Goyal A., Gupta V.K. and Anad J.D., 1994, Z. Phys. A, 349, 93.

Haensel P., Zdunik J.L. and Schaefer R., 1986, A\&A, 160, 121.

Ho W.C.G. and Lai D., 2000, ApJ, 543, 386.

Jones P.B., 2001, Phys. Rev. Lett., 86, 1384.

Klimov V.V., 1982, Sov. Phys. JETP, 55, 199.

Kojima Y., 1998, MNRAS, 293, 423.
Kokkotas K.D. and Stergioulas N., 1999, A\&A, 341, 110.

Lattimer J.M., van Riper K.A. and Prakash M., 1994, ApJ, 425, 802.

Lindblom L., Owen B.J. and Morsink S.M., 1998, Phys. Rev. Lett., 80, 4843.

Lindblom L. and Mendell G., 2000, Phys. Rev. D, 61, 104003.

Lindblom L., Owen B.J. and Ushomirsky G., 2000, Phys. Rev. D, 62, 084030.

Lindblom L., Mendell G. and Owen B.J., 1999, Phys. Rev. D, 60, 064006.

Lindblom L., Toline J.E. and Vallisneri M., 2001, Phys. Rev. Lett., 86, 1152.

Madsen J., 1992, Phys. Rev. D, 46, 3290

Madsen J., 1998, Phys. Rev. Lett., 81, 3311

Madsen J., 2000, Phys. Rev. Lett., 85, 10

Mendell G., 2001, Phys. Rev. D, 64, 044009.

Owen B.J. et al. 1998, Phys. Rev. D, 58, 084020.

Page D., Prakash M., Lattimer J.M. and Steiner A.W., 2000, Phys. Rev. Lett., 85, 2048.

Pisarski R.D., 1989, Nucl. Phys. A, 498, 423c.

Rezania V. and Maartens R., 2000, Phys. Rev. Lett., 84, 2560.

Rezzolla L., Lamb F.K. and Shapiro S.L., 2000, ApJ, 531, 139.

Sawyer R.P., 1989, Phys. Lett, B 233,412

Schertler K., Greiner C. and Thoma M.H., 1997, Nucl. Phys, A, 616, 659.

Spruit H.C., 1999, A\&A, 41, L1.

Wang Q.D. and Lu T., 1984, Phys. Lett. B, 148, 211.

Weldon H.A., 1982, Phys. Rev. D, 26, 1394 and 2789 .

Witten E., 1984, Phys. Rev. D, 30, 72.

Vija H. and Thoma M.H., Phys. Lett. B, 342, 212. 
Zheng X.P., Yang S.H., Li J. R. and Cai X. 2002, Phys. Lett. B, 548, 29.

This 2-column preprint was prepared with the AAS LATEX macros v5.0. 


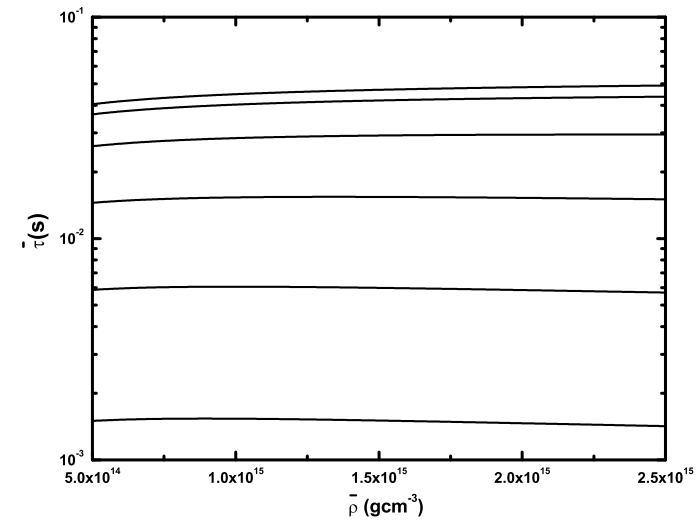

Fig. 1. - The time scales $\bar{\tau}_{B}$ as functions of mass density for different couplings $g$, which are $0,1,2$, $3,4,5$ respectively from top to bottom 


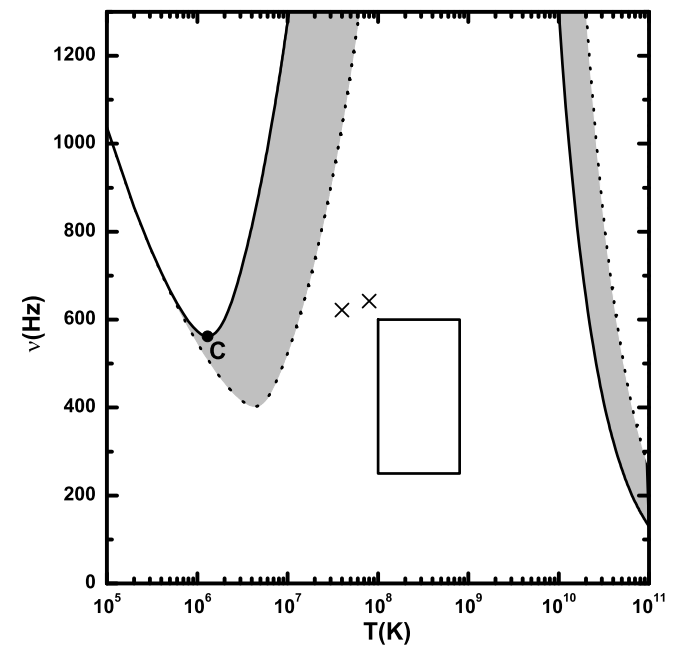

Fig. 2.- Critical spin frequencies for strange stars as functions of temperature. The lower contour(dotted) curves stand for free quark gas. The shadow displays the medium effect. 


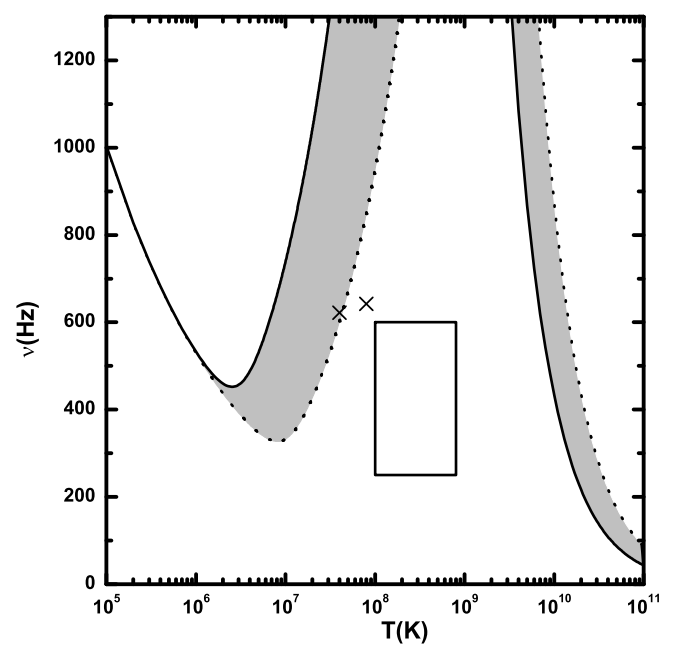

Fig. 3.- As Fig 2, but assuming a 2SC state. 


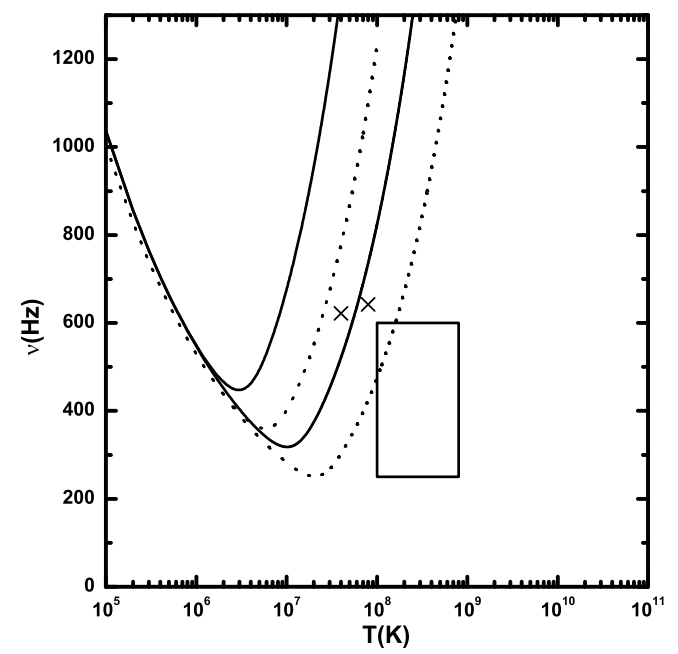

Fig. 4. - The r-mode instability windows for the case of $m_{s}=100 \mathrm{MeV}$. The solid curves denote the strange stars with normal phase and the dashed curves represent the case with $2 \mathrm{SC}$ phase. The lower solid and dashed curves correspond to the case of non-interacting quark matter. 